\title{
Investigation of an energy nonadditivity for nonextensive systems
}

\author{
WANG $\mathrm{Ru}^{1,2^{*}}$, NGANSO DJANTENG Sandrine ${ }^{1}$, KAABOUCHI EI Aziz ${ }^{1} \&$ \\ WANG Qiuping Alexandre ${ }^{1,3^{*}}$ \\ ${ }^{1}$ Institut Supéieur des Matériaux du Mans, 44, Avenue F. A. Bartholdi, Le Mans 72000, France; \\ ${ }^{2}$ College of Information Science and Engineering, Huaqiao University, Quanzhou 362021, China; \\ ${ }^{3}$ LPEC, UMR CNRS 6087, Université du Maine, Av. O. Messiaen, Le Mans 72085, France
}

Received March 26, 2011; accepted May 8, 2011

\begin{abstract}
We investigate the energy nonadditivity relationship $E(A \cup B)=E(A)+E(B)+\alpha E(A) E(B)$ which is often considered in the development of the statistical physics of nonextensive systems. It was recently found that $\alpha$ in this equation was not constant for a given system in a given situation and could not characterize nonextensivity for that system. In this work, we select several typical nonextensive systems and compute the behavior of $\alpha$ when a system changes its size or is divided into subsystems in different fashions. Three kinds of interactions are considered. It is found by a thought experiment that $\alpha$ depends on the system size and the interaction as expected and on the way we divide the system. However, one of the major results of this work is that, for given system, $\alpha$ has a minimum with respect to division position. Around this position, there is a zone in which $\alpha$ is more or less constant, a situation where the sizes of the subsystems are comparable. The width of this zone depends on the interaction and on the system size. We conclude that if $\alpha$ is considered approximately constant in this zone, the two mathematical difficulties raised in previous studies are solved, meaning that the nonadditive relationship can characterize the nonadditivity of the system as an approximation. In all the cases, $\alpha$ tends to zero in the thermodynamic limit $(N \rightarrow \infty)$ as expected.
\end{abstract}

nonextensive system, nonadditive energy, nonadditivity parameter

Citation: Wang R, Nganso Djanteng S, Kaabouchi Ei A, et al. Investigation of an energy nonadditivity for nonextensive systems. Chinese Sci Bull, 2011, 56: 3661-3665, doi: 10.1007/s11434-011-4676-8

Many kinds of physical systems in nature are recognized as energy nonadditive or nonextensive ${ }^{1)}$. This includes small size systems as well as large size systems having long range interaction such as gravity. For these systems, knowing the nonadditivity or nonextensivity in energy and entropy will be very useful for understanding some physical properties and for establishing corresponding theoretical descriptions. Energy and entropy additivity is a crucial hypothesis and a cornerstone for many physical theories [1]. How to replace this basic hypothesis is obviously a puzzling problem. In view of the diversity of the nonadditive systems in nature $[2,3]$, it is unlikely that a universally valid and simple relationship exists.

In this work, we focus on a special relationship of energy nonadditivity given by

$$
E(A \cup B)=E(A)+E(B)+\alpha E(A) E(B),
$$

where $A$ and $B$ are two subsystems of a composite system $A \cup B$ and $E$ is their energy. $\alpha$ characterizes the energy nonadditivity of the system under consideration and should be constant for a given system in a given situation with constant $E(A \cup B)$. The same logic can be found in [4] for a similar nonadditivity in entropy with many examples of empirically determined $\alpha$ for entropy.

This relationship is frequently considered in the development of statistical physics for nonextensive systems [5-8]. Ou et al. used eq. (1) as a first principle to show that the $q$-exponential distribution [8] can occur in small systems

*Corresponding authors (email: wangr@ismans.fr, awang@ismans.fr)

1) The readers should be careful with the two notions of nonadditivity and nonextensivity. They are not always equivalent to each other. 
and gave an example with the potential energy of spherical gravitation systems [5]. In calculating eq. (1), they noticed that $\alpha$ could not be constant for a given gravitational system. Further analysis was made in [9] where it was found that, apart from the problem of changing $\alpha$ for a given system with given physical conditions, another mathematical paradox arises when a combination of more than two subsystems is attempted. The conclusion is that, a priori, $\alpha$ cannot characterize the nonadditive property of a system because it may have an infinite number of values even when the system is in a completely given physical situation.

Given the result of [9], we are now interested in knowing how $\alpha$ changes as a function of the division position, what would be the result with interactions other than gravity, and whether eq. (1) can be eventually used as an approximation in some special cases. We computed the behavior of $\alpha$ for three typical nonextensive systems: a gravitational sphere, a two-dimensional lattice with periodical interaction and a two-dimensional lattice with interacting classical rotators. The result is, on the one hand, that the analysis of [9] is generally confirmed, and on the other hand, that $\alpha$ has a minimum with respect to the division position. Around the minimal $\alpha$, there is a zone of very slow variation in $\alpha$. The width of this zone depends on the interaction and on the system size. $\alpha$ can be considered as approximately constant in this zone depending on the required precision in energy. This implies that eq. (1) can be a good approximation for characterizing the nonadditivity of energy of some systems.

\section{Self-gravitation potential energy}

The nonextensive energy of the self-gravitation potential was calculated in [5]. As shown in Figure 1, the total mass $M$ is isotropically distributed in the system with the mass density $\rho$, and we can easily write the gravitational potential energy of such system as

$$
V_{\text {total }}=\int_{0}^{R} \frac{G\left(\rho \frac{4}{3} \pi r^{3}\right) \mathrm{d} m}{r},
$$

where $G$ is the gravitational constant and

$$
\mathrm{d} m=\rho 4 \pi r^{2} \mathrm{~d} r .
$$

Substituting eq. (3) into eq. (2) yields

$$
V_{\text {total }}=G \rho^{2} \frac{(4 \pi)^{2}}{3} \int_{0}^{R} \frac{r^{5} \mathrm{~d} r}{r}=G \rho^{2} \frac{(4 \pi)^{2}}{3} \frac{R^{5}}{5} .
$$

This system is separated into two parts as shown in Figure 1 . The potential energy of part 1 is given by

$$
V_{1}=G \rho^{2} \frac{(4 \pi)^{2}}{3} \int_{0}^{R_{1}} \frac{r^{5} \mathrm{~d} r}{r}=G \rho^{2} \frac{(4 \pi)^{2}}{3} \frac{R_{1}^{5}}{5},
$$

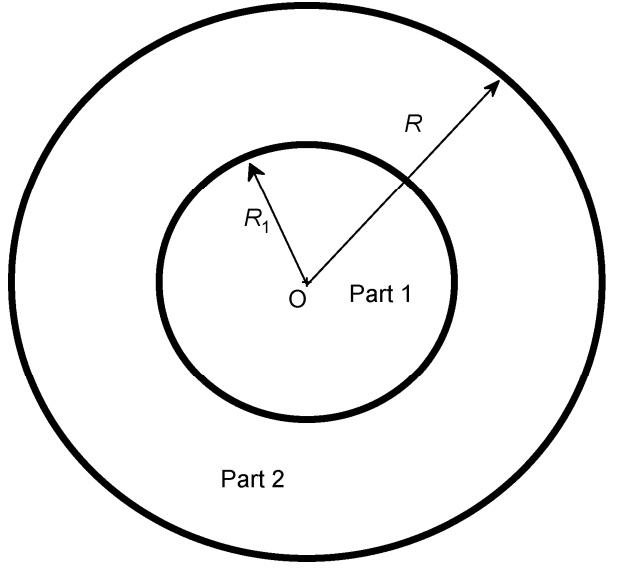

Figure 1 Spherical self-gravitation system is separated into two subsystems by a spherical surface with radius $R_{1}$.

while the potential energy of part 2 is given by

$$
\begin{aligned}
V_{2} & =G \rho^{2} \frac{(4 \pi)^{2}}{3} \int_{R_{1}}^{R} \frac{\left(r^{3}-R_{1}^{3}\right) r^{2} d r}{r} \\
& =G \rho^{2} \frac{(4 \pi)^{2}}{3}\left(\frac{R^{5}}{5}-\frac{R_{1}^{3} R^{2}}{2}+\frac{3 R_{1}^{5}}{10}\right) .
\end{aligned}
$$

From eqs. (4)-(6) one can get

$$
V_{\text {total }}=V_{1}+V_{2}+V_{1} V_{2}\left(G \rho^{2} \frac{(4 \pi)^{2}}{3}\right)^{-1} \frac{25}{R^{5}} \frac{p^{-2}-1}{2-5 p^{3}+3 p^{5}},
$$

where $p=R_{1} / R$, and the third term in the right hand side of eq. (7) implies that the potential energy of a self-gravitating system is nonadditive because

$$
\begin{aligned}
\alpha & =\left(G \rho^{2} \frac{(4 \pi)^{2}}{3}\right)^{-1} \frac{25}{R^{5}} \frac{p^{-2}-1}{2-5 p^{3}+3 p^{5}} \\
& =\left(G \rho^{2} \frac{(4 \pi)^{2}}{3}\right)^{-1} \frac{25}{R_{1}^{5}} \frac{p^{-2}-1}{2 p^{-5}-5 p^{-2}+3} \neq 0,
\end{aligned}
$$

unless $1 / p \rightarrow \infty$ for given $R_{1}$. In Figure 2(a), we show the variation in $\alpha$ versus $p$ for given total radius $R$. The typical self-gravitation energy of the earth is considered, $G=6.674 \times$ $10^{-11} \mathrm{~N} \cdot \mathrm{m}^{2} / \mathrm{kg}^{2}$ and $\rho_{\text {earth }}=5.515 \times 10^{3} \mathrm{~kg} / \mathrm{m}^{3}$. It is found that $\alpha$ first decreases with increasing $p$ and then increases. From eq. (7), it is easy to deduce that the minimum value of $\alpha$ is located at $p \approx 0.748$ for any given $R$ and that $\alpha$ becomes infinite at $p=0$ and $p=1$ (note that the masses of the two parts are equal when $p \approx 0.794$ ). Figure $2(\mathrm{~b})$ shows the variation in $\alpha$ versus $R$ for three values of $p: p=0.2,0.4,0.6$. For all these three cases, $\alpha \rightarrow 0$ as $R \rightarrow \infty$. It is found that, around the minimum value $\alpha_{\text {min }}$, there is a zone in which $\alpha$ varies slowly when $R$ is sufficiently large. In this zone, $\alpha$ may be considered as constant depending on the precision in $\alpha$ or in energy we want to reach. For example, if we consider $\alpha$ as constant when its variation $\left|\alpha-\alpha_{\min }\right|$ is smaller than a bound, 

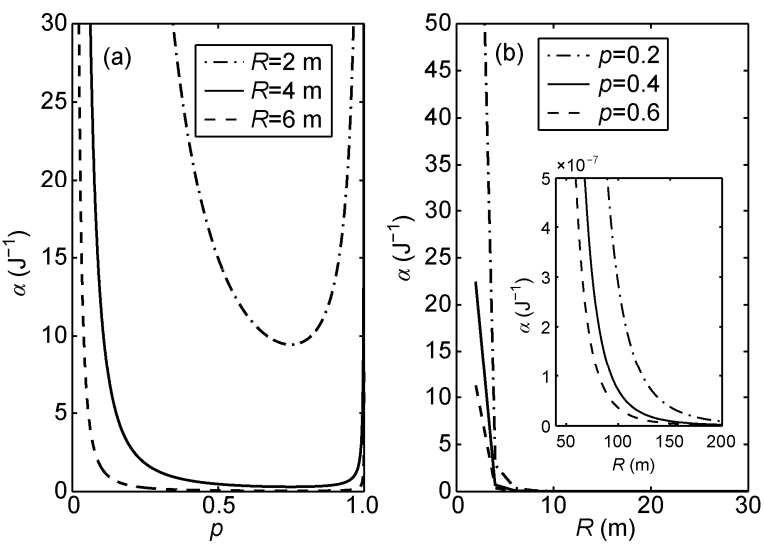

Figure 2 (a) Variation in $\alpha$ as a function of relative cutting position $p$ for a given radius $R$; (b) variation in $\alpha$ versus $R$ for $p=0.2,0.4,0.6$. Inset shows the zoomed in region of $\alpha<5 \times 10^{-7} \mathrm{~J}^{-1}$

we can define an interval of $p$ around $\alpha_{\min }$ as follows: $\Delta p=$ $p_{\text {up }}-p_{\text {low }}$ where $p_{\text {up }}$ (or $p_{\text {low }}$ ) is the upper (or lower) bound of $p$ beyond (or below) $p\left(\alpha_{\text {min }}\right)$. Of course, we can reach any precision for eq. (1) to be a good approximation in this interval. The larger the precision is, the smaller is the interval. For a given precision, the larger the system $(R)$ is, the flatter the variation in $\alpha$ around $\alpha_{\min }$, and the larger $\Delta p$. This is shown in Figure 3(a) for $\left|\alpha(R, p)-\alpha_{\min }(R, p)\right| \leqslant 10^{-6}$. One sees that the width $\Delta p$ increases with $R$ and approaches the maximum (unity) in the thermodynamic limit. If one uses the relative variation $\left|\alpha-\alpha_{\min }\right| / \alpha_{\min }$ as the precision criterion, $\Delta p$ will be independent of $R$. For each precision, there exists a corresponding interval $\Delta p=p_{\text {up }}-p_{\text {low. }}$. As Figure 3(b) shows, the relative variation interval of $\Delta p$ increases as the precision decreases.
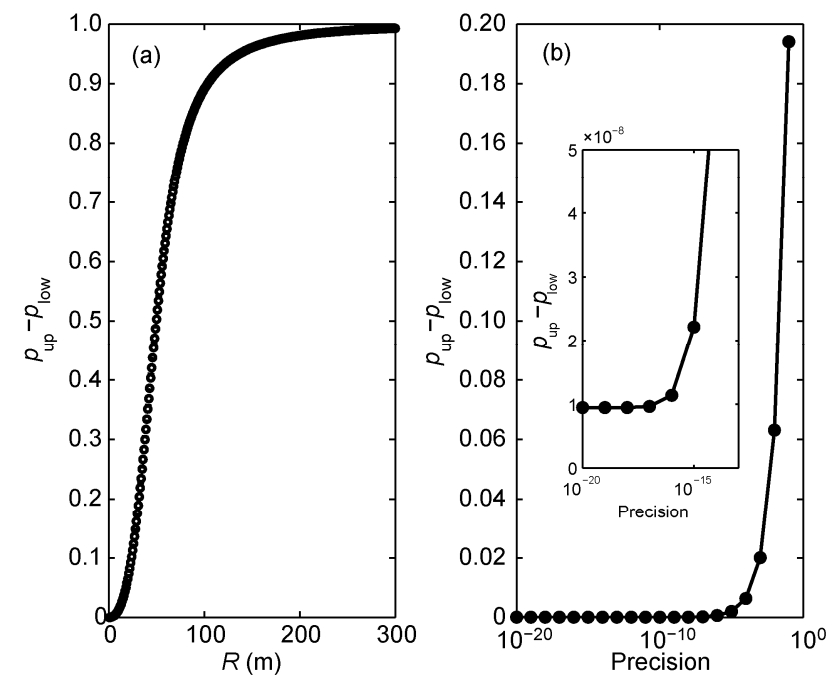

Figure 3 (a) Variation in the interval $\Delta p=p_{\text {up }}-p_{\text {low }}$ around $p\left(\alpha_{\text {min }}\right)$ as a function of the total radius $R$ of the gravitational sphere for the case $\left|\alpha-\alpha_{\min }\right| \leqslant 10^{-6}$; (b) variation in the interval $\Delta p=p_{\text {up }}-p_{\text {low }}$ as a function of precisions defined as the relative variation $\left|\alpha-\alpha_{\min }\right| / \alpha_{\min }$. Inset shows the zoomed in region of $\Delta p<5 \times 10^{-8}$.

\section{Two-dimensional lattice with periodical interaction}

The periodic potential is often considered in solid state physics. Here, we consider a two-dimensional square lattice. The potential energy of the system is defined by

$$
V=\frac{1}{2} \sum_{(i, j)} \sin \left(\frac{2 \pi r_{i j}}{\lambda}\right),
$$

where $\lambda$ is the periodic length and $r_{i j}$ is the distance between the particles $i$ and $j$. The length for each lattice cell is one unit. The sum runs over all $N$ particles. This system is divided into two parts as shown in Figure 4. The relative cutting position is defined by $p=L_{\mathrm{l}} / L$ where $L_{\mathrm{l}}$ is the horizontal length of the left part and $L$ is the total side length of the lattice. The potential energies of the two parts are respectively

$$
V_{1}=\frac{1}{2} \sum_{(i, j) \in \text { part } 1} \sin \left(\frac{2 \pi r_{i j}}{\lambda}\right)
$$

and

$$
V_{2}=\frac{1}{2} \sum_{(i, j) \in \text { part } 2} \sin \left(\frac{2 \pi r_{i j}}{\lambda}\right) .
$$

The variation in $\alpha=V-V_{1}-V_{2} / V_{1} V_{2}$ versus $p$ and periodic length $\lambda$ is shown in Figure 5. The absolute value of $\alpha$ decreases with increasing $p$ for small $p$ and increases for large $p$, and oscillates in between small and large $p$. This oscillation is a consequence of the periodic property. A larger system and shorter periodic length will lead to smaller oscillation.

Due to the symmetry of the system, $\alpha$ is symmetrical at $p=0.5$ and becomes a maximum when $p \rightarrow 1$ and $p \rightarrow 0$. We plotted $\alpha_{p=0.5}$ as a function of the system size for different

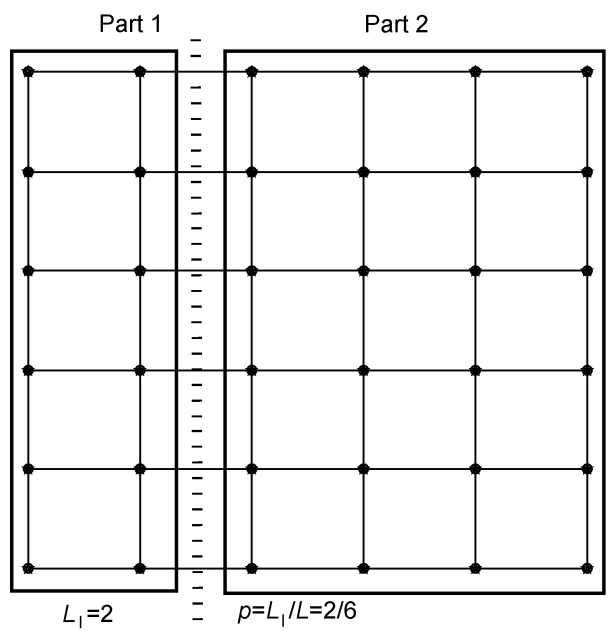

Figure 4 Two-dimensional lattice is separated into two subsystems with relative cutting position $p=2 / 6$. 

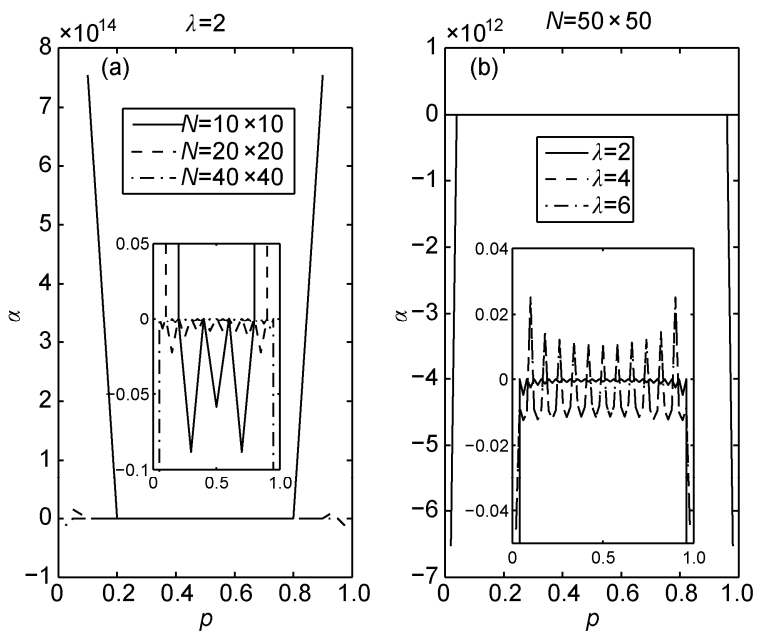

Figure 5 (a) Variation in $\alpha$ as a function of relative cutting position $p$ for different particle numbers and a given $\gamma$, and (b) for different $\gamma$ with a given particle number. Inset shows the zoomed in region of oscillation.

periodic lengths in Figure 6, and found that, as expected, $\alpha$ will tend to 0 as the system size increases.

\section{A two-dimensional lattice of interacting classical rotators}

The potential energy of classical interacting planar rotators on a two-dimensional lattice is defined as

$$
V=\sum_{(i, j)} \frac{1-\cos \left(\theta_{i}-\theta_{j}\right)}{r_{i j}^{\gamma}},
$$

where $r_{i j}$ runs over all possible distances between two rotators within the two-dimensional lattice. The length for each lattice cell is one unit. The particular case $\gamma=0$ is referred to in the literature as the HMF model, and has been intensively studied in the last decade [10]. In this specific situation, each site carries a rotator which can either be in one direction $(\theta)$ or be in the opposite $(\theta+\pi)$ direction. Each rotator

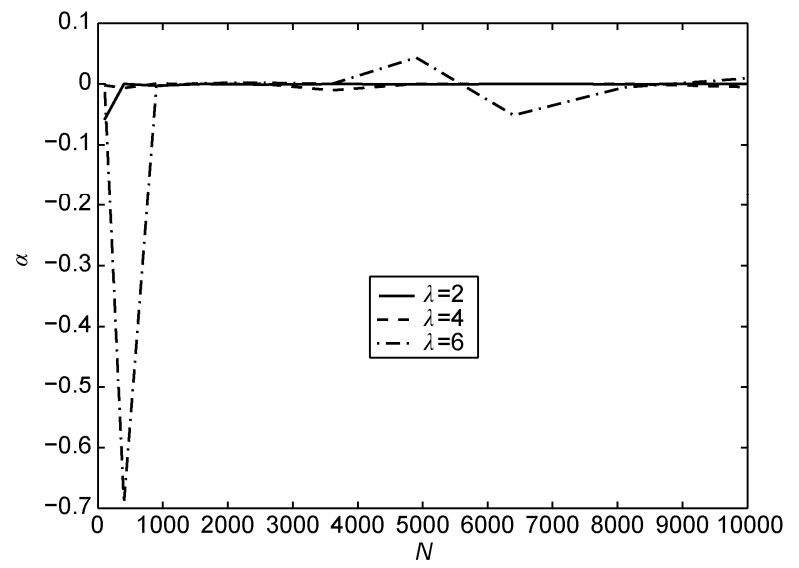

Figure $6 \alpha_{p=0.5}$ as a function of $N$ for different $\lambda$. has the opposite direction to its nearest four neighbors, as shown in Figure 7.

This system is divided into two parts with the same definition of relative cutting position $p$. The variation in $\alpha$ as a function of $p$ for different system sizes $N$ and for different $\gamma$ are shown in Figure 8. Due to the symmetry of the system, $\alpha$ is symmetrical with respect to $p=0.5$. As in the two systems described above, $\alpha$ decreases with $p$ at first and then increases. The minimal value of $\alpha$ is located at $p=0.5$. The larger $N$, the smoother the variation in $\alpha$ around $\alpha_{\min }$. However, all other things being equal, $\alpha$ is not a monotonically decreasing function of $\gamma$ (Figure 8(b)). The variation in $\alpha_{\min }$ as a function of $\gamma$ is shown in Figure 9(b) for different system sizes $N$. We found that $\alpha_{\min }$ reaches the maximum value when $\gamma \approx 1.5$ for all $N$. Likewise, $\alpha_{\min }$ as a function of system size $N$ for different $\gamma$ is shown in Figure 9(a). Note that $\alpha \rightarrow 0$ in the thermodynamic limit $(N \rightarrow \infty)$.

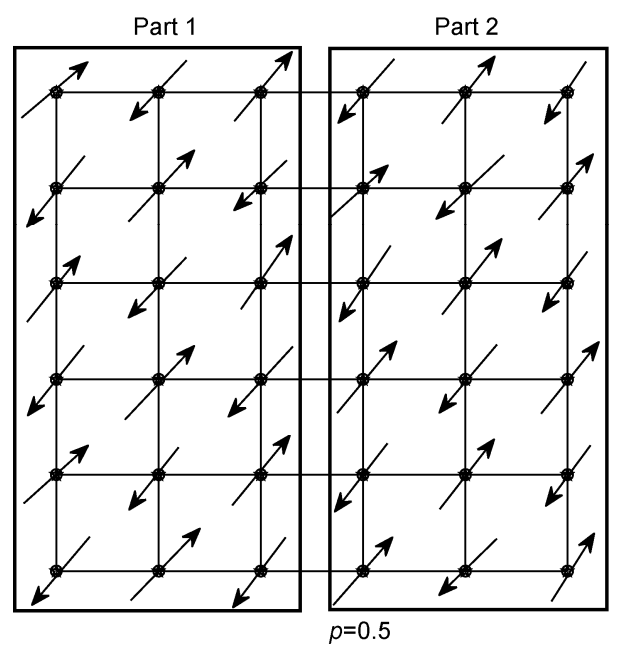

Figure $7 \quad N$ interacting classical rotators on two-dimensional lattice with relative cutting position $p=0.5$.
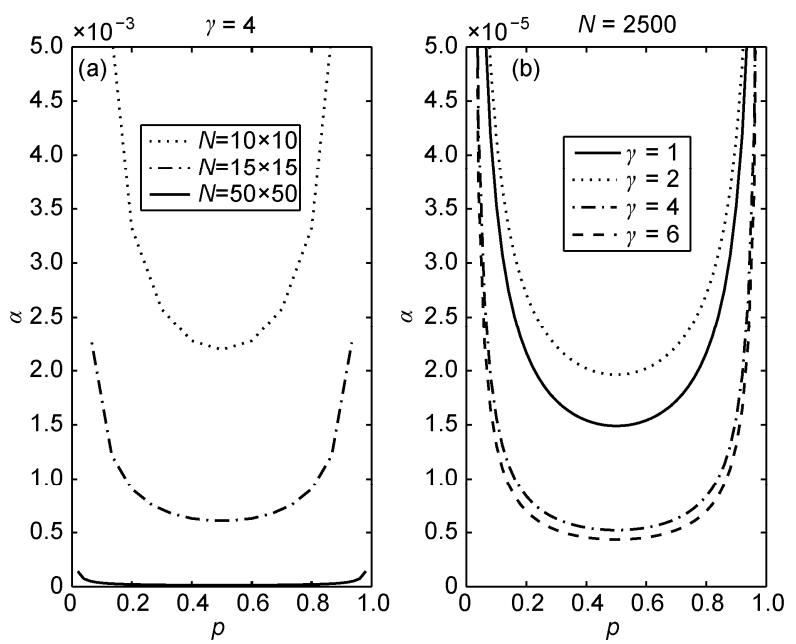

Figure $8 \alpha$ as a function of relative cutting position $p$ for different system sizes $N$ (a) and different $\gamma(\mathrm{b})$. 

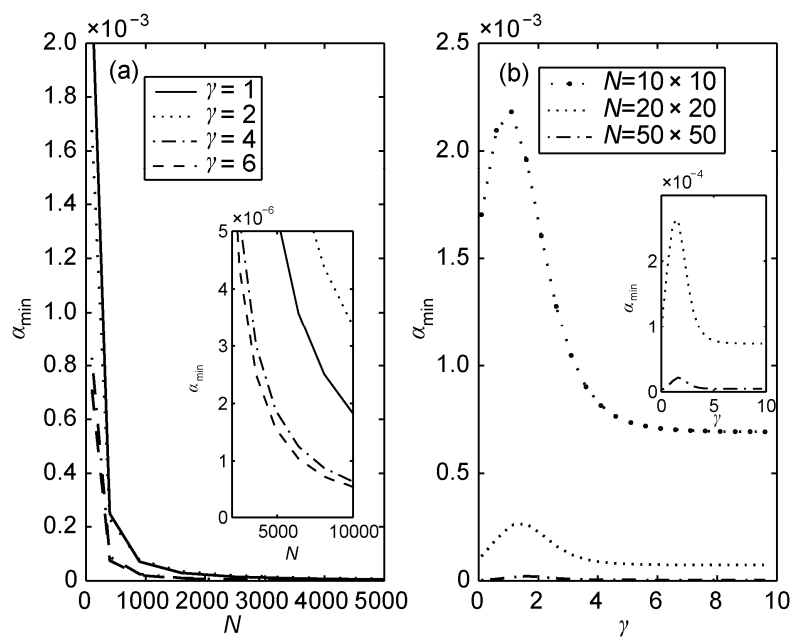

Figure 9 (a) $\alpha_{\min }$ (for $p=0.5$ ) as a function of $N$ for different $\gamma$. Inset shows the zoomed in region of $\alpha_{\min }<5 \times 10^{-6}$. (b) $\alpha_{\min }$ (for $p=0.5$ ) as a function of $\gamma$ for different $N$. Inset shows the zoomed in region of $\alpha_{\min }<3 \times 10^{-4} . \alpha_{\text {min }}$ reaches the maximum value when $\gamma \approx 1.5$ for all $N$.

\section{Concluding remarks}

The results of this work confirm the mathematical analysis given in [9], i.e. $\alpha$ in the expression $E(A \cup B)=E(A)+E(B)+$ $\alpha E(A) E(B)$ for energy $E$ is not constant for a given system in a given physical situation with constant $E(A \cup B)$. It depends on the way we divide the system (note that the division can be made in a thought experiment). Hence from a theoretical point of view, eq. (1) is not an exact physical relationship with $\alpha$ characterizing the nonadditivity of the system, as indicated in [9].

However, the numerical calculation of the behavior of $\alpha$ as a function of the division position of the three considered systems revealed an interest aspect on the variation in $\alpha$ : for a given system in a completely given situation, $\alpha$ has a minimum with respect to division position. Around this position, there is a zone of division positions in which $\alpha$ varies very slowly so that it is possible to consider it as constant. This zone of division corresponds to the case where the sizes of the two subsystems are comparable. The width of this zone depends on the interaction, on the system size, and obviously on the precision in energy we want to reach in the application. We can reach any precision by reducing the width of that zone.

When $\alpha$ is considered as approximately constant in this zone, the two mathematical difficulties raised in [9] will be solved, meaning that the nonadditive relationship given by eq. (1) can characterize the nonadditivity of at least potential energy as an approximation in a limited zone of division of the total system.

As expected, $\alpha$ always tends to zero in the thermodynamics limit $(N \rightarrow \infty)$.

We thank T. Plassier, A. Ferrer, G. Drapeau, C. Chantoiseau, R. Chang for their contributions to the simulation programs. This work was supported by the Region des Pays de la Loire of France (2009-9397) and Research Foundation of HuaQiao University (09BS511).

1 Terletskii Y P. Statistical Physics. Amsterdam: North-Holland, 1971

2 Ha M, Li Y, Li J, et al. The key theorem and the bounds on the rate of uniform convergence of learning theory on Sugeno measure space. Sci China Ser F-Inf Sci, 2006, 49: 372-385

3 Liu S, Yang X, Yang Z. Modification of free-energy density functional theory approach for prediction of high-pressure mixture adsorption. Chinese Sci Bull, 2008, 53: 1358-1364

4 Tsallis C. Nonadditive entropy and nonextensive statistical mechanics-An overview after 20 years. Braz J Phys, 2009, 39: 337-356

$5 \mathrm{Ou} \mathrm{C} \mathrm{J,} \mathrm{Li} \mathrm{W,} \mathrm{Tsobnang} \mathrm{F,} \mathrm{et} \mathrm{al.} \mathrm{Possible} \mathrm{canonical} \mathrm{distributions} \mathrm{for}$ finite systems with nonadditive energy. Physica A, 2008, 387: 57615767

6 Wang Q A, Nivanen L, Mehaute A Le, et al. Temperature and pressure in nonextensive thermostatistics. Europhys Lett, 2004, 65: 606-612

7 Li W, Wang Q A, Nivanen L, et al. On different q-systems in nonextensive thermostatistics. Eur Phys J B, 2005, 48: 95-100

8 Tsallis C, Tirnakli U. Nonadditive entropy and nonextensive statistical mechanics-Some central concepts and recent applications. J Phys Conf Ser, 2010, 201: 1-15

9 Wang Q A, Ou C J, Chen J C. The q-nonadditivity of nonextensive statistics is not a true physical property. arXiv: 0910.3826v2

10 Chavanis P H, Campa A. Inhomogeneous Tsallis distributions in the HMF model. Eur Phys J B, 2010, 76: 581-611

Open Access This article is distributed under the terms of the Creative Commons Attribution License which permits any use, distribution, and reproduction in any medium, provided the original author(s) and source are credited. 